\title{
FNAL BOOSTER: EXPERIMENT AND MODELING
}

\author{
P. Spentzouris*, J. Amundson, FNAL, Batavia, IL 60510, USA
}

\begin{abstract}
We present measurements of transverse and longitudinal beam phase space evolution during the first two hundred turns of the FNAL Booster cycle. We discuss the experimental techinque, which allowed us to obtain turn-by-turn measurements of the beam profile. The experimental results are compared with the prediction of the Synergia 3D space charge simulation code.
\end{abstract}

\section{INTRODUCTION}

The Fermilab Booster is a rapid-cycling, $15 \mathrm{~Hz}$, alternating gradient synchrotron with a radius of 75.47 meters. The lattice consists of 96 combined function magnets in 24 periods, with nominal horizontal and vertical tunes of 6.7 and 6.8 respectively. The Booster accelerates protons from a kinetic energy of $400 \mathrm{MeV}$ to $8 \mathrm{GeV}$, at a harmonic number $\mathrm{h}=84$, using $17 \mathrm{rf}$ cavities with frequency which slews between $37.7 \mathrm{MHz}$ (at injection) and $52.8 \mathrm{MHz}$ (at extraction). The revolution time at injection is $2.2 \mu \mathrm{s}$. A comprehensive technical description of the Booster as built can be found in reference [1]. The injection system utilizes the $\mathrm{H}^{-}$charge-exchange injection technique. The typical linac peak-current is $45 \mathrm{~mA}$; usually up to eleven turns of $\mathrm{H}^{-}$beam are injected in the booster. The injected beam is a stream of bunches equally spaced at the linac rf frequency of 201.2 MHz. During injection, a pulsed orbit bump magnet system (ORBUMP) is used to superimpose the trajectories of the circulating (protons) and injected $\left(\mathrm{H}^{-}\right)$beams.

There are many factors affecting the behavior of the Booster beam, including the energy and emittance of the incoming beam, nonlinear field errors and space charge effects, which are believed to be responsible for a significant fraction of the observed losses in the Booster [2] during the first $2 \mathrm{~ms}$ of the cycle (injection, capture, and bunching phase). Since the performance of the Booster is what makes or breaks the FNAL neutrino program, and its stable operation is required for the FNAL collider program, it is essential to study and quantify these effects. In order to achieve this goal, we have developed a fully three dimensional (3D), Particle In Cell (PIC) model of the booster, based on the package Synergia [3]. The Synergia package has been developed under the DOE SciDAC initiative for accelerator modeling, Advanced Computing for $21^{\text {st }}$ Century Accelerator Science and Technology. Synergia incorporates existing packages for modeling 3D space charge and computing transfer maps using Lie algebraic techniques. It utilizes a split operator technique for particle propagation, includes a parser of the Methodical Acceler-

\footnotetext{
*spentz@fnal.gov
}

ator Design (MAD) language, and has multi-turn injection modeling capabilities. The code has the capability to compute higher order transfer maps, but linear maps were used for the simulations presented in this paper.

In the following sections, we describe measurements of Booster beam evolution during the first 200 turns after injection, and present comparisons with the Synergia model.

\section{EXPERIMENTAL DATA AND COMPARISON TO SIMULATION}

The objective of the experiments was to study the beam evolution in the first few hundred turns after injection, by comparing transverse and longitudinal beam widths to the simulation, with single turn time resolution, for different beam currents. The FNAL Booster has two measuring devices capable of measuring of beam widths with such resolution: the Ion Profile Monitor detector (IPM) [4], which utilizes the ions from ionization of the residual gas by the proton beam to measure transverse beam profiles, and the Resistive Wall Monitor (RWM) device, which utilizes the induced current on the beam pipe by the particle beam, to measure the longitudinal beam profile. Since the response of the IPM depends on the charge of the beam, and since the goal of the experimental program is to use this detector for a quantitative study of space charge effects, we installed a third measuring device, the "flying beam" wire, in order to check and calibrate the performance of the IPM.

\section{IPM calibration}

The IPM detector measures the projection of the beam distribution on each one of the transverse coordinates. An applied transverse clearing field of $8 \mathrm{kV}$ causes the ions to drift to a micro-channel plate (MCP). (The beam direction defines the longitudinal coordinate). The detector is $0.5 \mathrm{~m}$ long, with a transverse gap of $12 \mathrm{~cm}$. The MCP plate is $8 \times 10 \mathrm{~cm}^{2}$ and has strip spacing $1.5 \mathrm{~mm}$. For an ideal measurement of one projection, the ions' drift should be parallel to the other (non-measured) coordinate. The external clearing field is applied on the beam along the non-measured coordinate to achieve this objective. The response of detector is complicated by the effect of the fields of the beam itself. The field due to the beam is not transverse, so it distorts the behavior from the ideal. As a result, the response of the IPM depends on the charge of the beam, so it has to be calibrated as a function of the injected number of protons.

In order to perform an experimental measure of the IPM calibration, we took width measurements simultaneously with the Booster IPM, the MI-8 extraction line wire cham- 
ber and the so-called "flying beam" wire [5]. The "flying beam" wire is a single wire measuring device at the Booster Long 1 section, which can be parked just outside the beam envelope of the injected beam, i.e beam envelope with the ORBUMP magnets on. At injection, the ORBUMP magnets keep the beam trajectory displaced by $\sim 4 \mathrm{~cm}$ with respect to the nominal beam orbit, so that the injected $\mathrm{H}^{-}$ions will pass through a stripping foil. The wire is placed between the displaced and nominal orbits. As the ORBUMP current decays, the beam sweeps through the wire, providing a measure of the horizontal beam profile. By recording the ORBUMP current as a function of time, $I(t)$, and the response of the wire as a function of time, $a(t)$, we can reconstruct the horizontal profile, $a(x)$, as measured by the wire by using the known beam position as a function of current, $x(I)$. The result of the calibration is a parameteri-

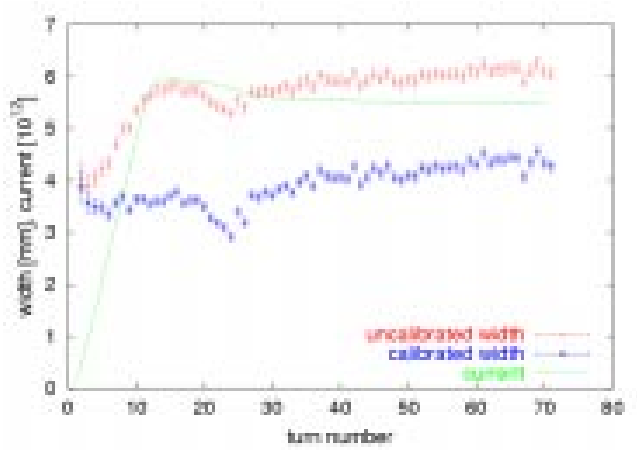

Figure 1: IPM horizontal beam width in the Booster ( $\mathrm{mm}$ ) as a function of time (in Booster turns -2.2 micro seconds), at injection. Both the calibrated (blue) and uncalibrated (red) widths are shown. Also shown (green curve) is a simultaneous measurement of the beam current in the machine, in units of $10^{12}$ protons/turn.

zation of the true width as a function of the measured width and the charge injected in the machine.

The importance of the calibration for the Booster IPM detector and the size of the beam self-field induced effects is demonstrated in Fig. 1, where both the calibrated and uncalibrated IPM beam profiles are shown, together with the beam current, as a function of time. The effect is most dramatic during the first eleven turns in the machine (injection time), since the beam current is changing. The change of the uncalibrated beam width clearly tracks the beam current change. The calibrated width shows a much smaller variation during the injection period.

\section{IPM transverse profile measurements}

We collected data with the IPM detector in three different time periods and for different configurations of the Booster injection bump magnets (ORBUMP). The different data sets were collected on November and December 2002, and April and May of 2003. We took measurements for different injected beam currents, for different timings of the injected beam with respect to the ORBUMP current pulse flattop, and for different shapes of the ORBUMP pulse (this change was implemented in 2003 to reduce heating problems). For each setting we took at least ten different measurements of beam profiles and beam currents over the full machine cycle. The data were corrected using the IPM calibration obtained with the procedure described in the previous section. There is a correlation between beam losses and beam envelope perturbations early in the cycle and the ORBUMP timing [6]. In Fig. 2 the horizontal and vertical beam widths are shown as a function of time, together with the normalized beam current. The different curves correspond to different injection timings with respect to the ORBUMP (averaging the corresponding data sets). All data sets were taken with $11 \times 40 \mathrm{~mA}$ of beam injected in the Booster. Both the beam width perturbations and the losses move in time with the change of timing with the same offset used in the trigger. One of the data sets averaged in
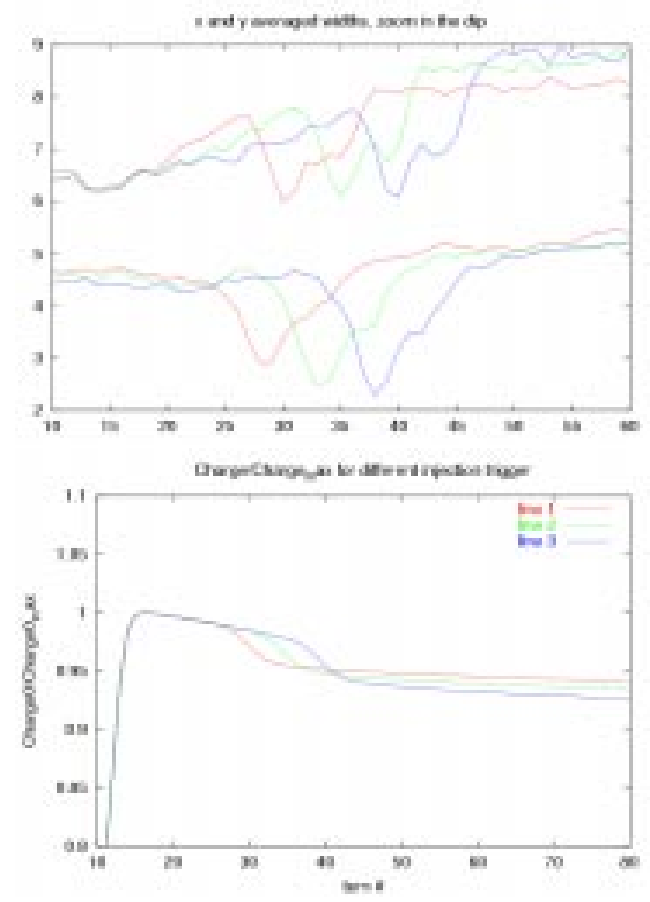

Figure 2: Top: IPM horizontal and vertical beam widths $(\mathrm{mm})$ versus time (turn number). Bottom: Normalized charge, to the maximum charge in the machine), versus time (turns). The time window is around the time that the ORBUMP turns off.

Fig. 2 is shown in Fig. 3 compared to the Synergia prediction (run with an input emittance two times smaller than the nominal). The model describes the data well, except of the region of the beam perturbation due to the injection bump, which was not included in the model. To verify that the injection bump fringe field can cause such effects we included a very simple model of this field in our simulation. A comparison of the prediction of the model to the data is shown in Fig. 4. The model includes just a quadrupole term in the non bending view of the bump magnet, with strength which has the same time dependence as the current pulse 


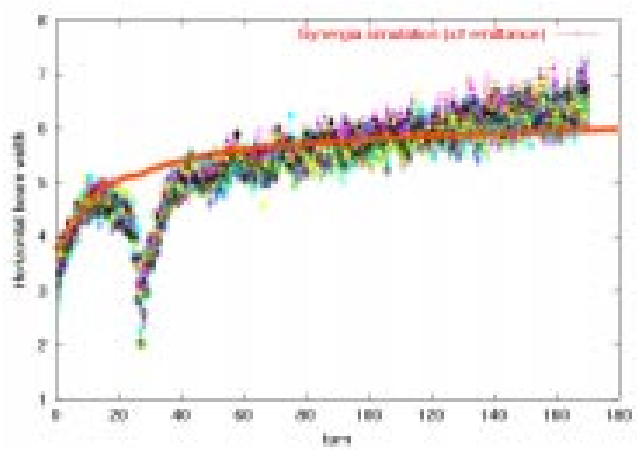

Figure 3: Comparison of horizontal beam width as a function of time, measured with the IPM, to the prediction of Synergia.

of the magnet. The model seems to reproduce the general

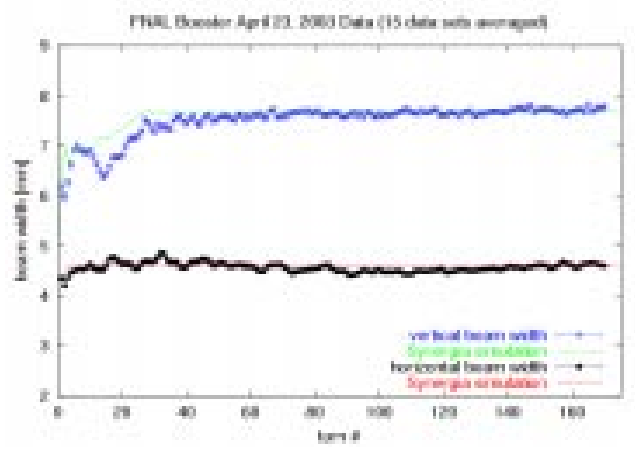

Figure 4: Synergia model, including the ORBUMP effect compared to horizontal and vertical beam widths as a function of time.

features of the data.

\section{Longitudinal profile measurements}

The purpose of these measurements were to obtain a data set under well defined conditions in order to check the model implementation. The data set was obtained with the Booster running DC (rf system off and no ramping of the magnet power supplies). This was done in order to simplify the running conditions and reduce the number of parameters in the comparisons. To further reduce complications in the initial conditions, only a single turn worth of Linac beam was injected in the machine. The current of the Linac beam was controlled by detuning one of the Linac quadrupoles. Under these conditions, we took data with injected beam of 11,20 , and $42 \mathrm{~mA}$, and recorded the evolution of the longitudinal beam profile using the RWM. Already, after $\sim 5$ turns in the machine, the beam distribution in time is almost flat within the $200 \mathrm{MHz}$ time slices (the injected beam has the structure of the $200 \mathrm{MHz}$ Linac rf system). In Fig. 5 the RMS of the time distribution of the beam in a $200 \mathrm{MHz}$ time slice is plotted versus the turn number. The data (points) are compared to the simulation (lines) for the different values of injected beam current.
There is good qualitative agreement between data and sim-

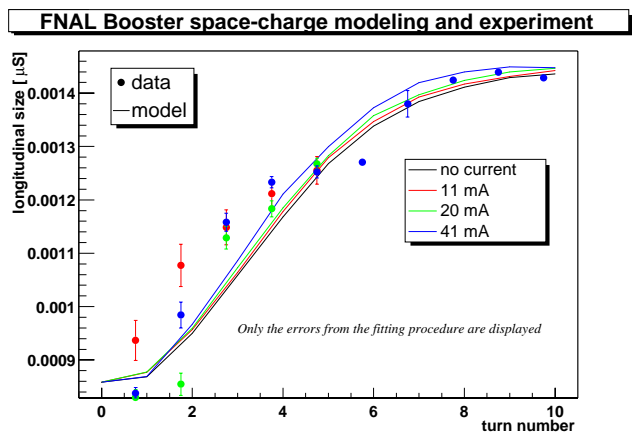

Figure 5: Longitudinal phase space evolution. Beam distribution RMS width in time as a function of turn number. The data from the RWM measurements (points with error bars) is compared to the model prediction from Synergia (lines).

ulation. The model predicts very small effects due to the different beam currents, well within the uncertainty of the measurement. Both the data and simulation extracted RMS widths tend to a constant value, since they are calculated within a $200 \mathrm{MHz}$ time slice. Note that in the simulation we only model one $200 \mathrm{MHz}$ bunch with periodic boundary conditions. This is an accurate representation of the main body of the beam (see discussion in [3]).

\section{REFERENCES}

[1] Booster Staff 1973 Booster Synchrotron ed E L Hubbard Fermi National Accelerator Laboratory Technical Memo TM405

[2] Popovic P and Ankenbrandt C 1998 Workshop on Space Charge Physics in High intensity Hadron Rings ed A U Luccio and W T Weng (Woodbury, New York: AIP Conference Proceedings) p 128

[3] J. Amundson and P. Spentzouris, "A Hybrid, Parallel 3D Space Charge Code with Circular Machine Modeling Capabilities", Proc. International Computational Accelerator Physics Conference (ICAP 2002), Michigan State University, Oct. 2002; see also J. Amundson and P. Spentzouris this proceedings

[4] Zagel J, Chen D, and Crisp J 1994 Beam Instrumentation Workshop (AIP Conference Proceedings 333) p 384

[5] J. Amundson, J. Lackey, P. Spentzouris, G. Jungman, and Linda Spentzouris, "Calibrating the Booster Ionization Profile Monitor: Preliminary Summary", FNAL Beams Document 495-v2, http://beamdocs.fnal.gov/, 2003.

[6] P. Spentzouris and J. Amundson "Booster beam profiles and losses before and after dog leg and ORBUMP magnet changes", FNAL Beams Document 581-v1, http://beamdocs.fnal.gov/, 2003. 